\title{
Mechanical Control of Periodic Precipitation in Stretchable Gels to Retrieve Information on Elastic Deformation and for the Complex Patterning of Matter
}

\author{
Mohammad Morsali, Muhammad Turab Ali Khan, Rahym Ashirov, Gábor Holló, \\ H. Tarik Baytekin, Istvan Lagzi, and Bilge Baytekin*
}

Material design using nonequilibrium systems provides straightforward access to complexity levels that are possible through dynamic processes. Pattern formation through nonequilibrium processes and reaction-diffusion can be used to achieve this goal. Liesegang patterns (LPs) are a kind of periodic precipitation patterns formed through reaction-diffusion. So far, it has been shown that the periodic band structure of LPs and the geometry of the pattern can be controlled by experimental conditions and external fields (e.g., electrical or magnetic). However, there are no examples of these systems being used to retrieve information about the changes in the environment as they form, and there are no studies making use of these patterns for complex material preparation. This work shows the formation of LPs by a diffusion-precipitation reaction in a stretchable hydrogel and the control of the obtained patterns by the unprecedented and uncommon method of mechanical input. Additionally, how to use this protocol and how deviations from "LP behavior" of the patterns can be used to "write and store" information about the time, duration, extent, and direction of gel deformation are presented. Finally, an example of using complex patterning to deposit polypyrrole by using precipitation patterns is shown as a template.

information of the chemical pathway of the NE phenomenon and therefore, have sensing and environment tracking abilities. Chemical patterns ${ }^{[4]}$ are "visual sums" of the complex events which take place in nonequilibrium chemical systems. Liesegang patterns (LPs) are one of these patterns ${ }^{[5]}$ formed by reaction-diffusion, which leads to periodic bands of precipitation. ${ }^{[6]}$ The formation of patterns is a transient nonequilibrium process driven mainly by reaction-diffusion. Since their discovery more than a century ago, ${ }^{[7]}$ LPs are one of the most studied patterns due to their wide occurrence in nature, ${ }^{[6,8,9]}$ and because of the straightforward monitoring of various chemical systems leading to these patterns in gel media. To control and model LP formation, parameters affecting the formation of LPs such as the concentrations of reagents, ${ }^{[10-12]}$ the gel concentrations, ${ }^{[13-15]}$ impurities in the gels, ${ }^{[13]}$ the degree of cross-linking in the gels, ${ }^{[16,17]}$ the $\mathrm{pH}$ of the media, ${ }^{[12,18]}$ electric fields, ${ }^{[18-21]}$

Material design using phenomena out of equilibrium, diffusion, and chemical patterns is currently a popular trend in material science since nonequilibrium (NE) phenomena give rise to emergent properties and higher levels of complexity. ${ }^{[1-3]}$ In a sense, materials formed during NE processes carry the

M. Morsali, M. T. A. Khan, R. Ashirov, Dr. H. T. Baytekin, Dr. B. Baytekin Chemistry Department

Bilkent University

Ankara 06800, Turkey

E-mail: b-baytekin@fen.bilkent.edu.tr

Dr. G. Holló, Dr. I. Lagzi

Department of Physics and BME-MTA Condensed Matter Physics

Research Group

Budapest University of Technology and Economics

Budapest $\mathrm{H}-1111$, Hungary

Dr. B. Baytekin

UNAM

Bilkent University

Ankara 06800, Turkey

The ORCID identification number(s) for the author(s) of this article can be found under https://doi.org/10.1002/adma.201905779.

DOI: 10.1002/adma.201905779 and microwave radiation ${ }^{[22]}$ have been investigated. Since Ostwald, ${ }^{[23]}$ many mathematical models have been developed to describe and model the reaction-diffusion phenomenon during LP formation using these results. ${ }^{[24-26]}$ Although there are numerous studies on the control of pattern formation and comments about the potentials of these structures ${ }^{[9]}$ for use in sensors, microelectromechanical systems, bioengineering, and microfluidics, ${ }^{[27]}$ there have been no studies that display a direct use for such periodic patterns in environment tracking or material design. Herein we show that the information embedded in the presence/absence, location, and geometry of the patterns formed in reaction-diffusion systems can be used to track mechanical changes in the surrounding environment and be used for the complex patterning of other (secondary) materials.

We propose such a connection between LP formation and the above mentioned "tracking" can be made by coupling a mechanical input to the reaction-diffusion process. It has been already shown that mechanical input is used to initiate and affect chemical reactions (referred to as mechanochemistry), ${ }^{[28,29]}$ chemically functionalize surfaces, ${ }^{[30,31]}$ and sometimes even alter conventional (thermal or photo) reaction pathways. ${ }^{[32]}$ In material science, there are also applications of macroscopic mechanical 
a)

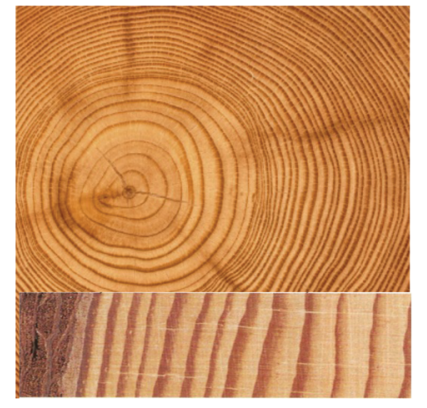

b)

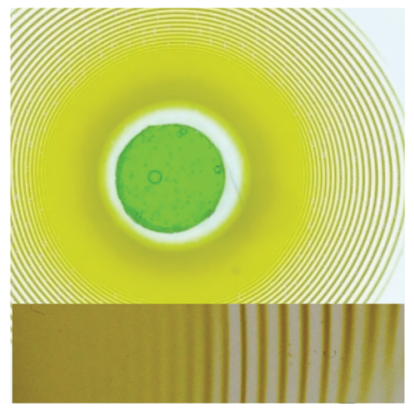

c)

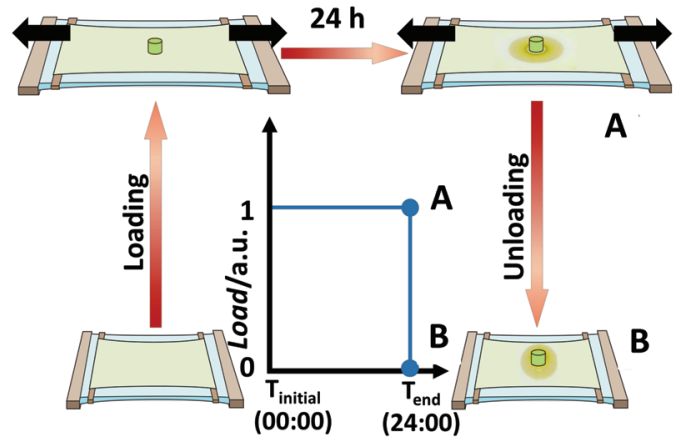

A:Stretched (loaded)

d)

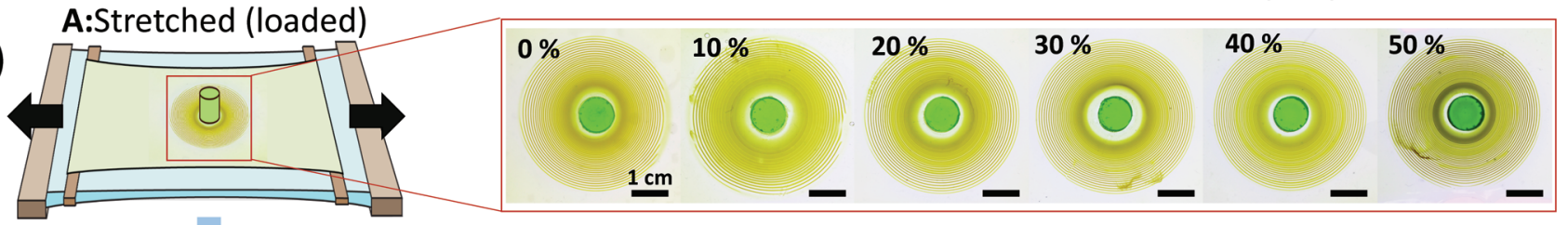

24 hours

e)

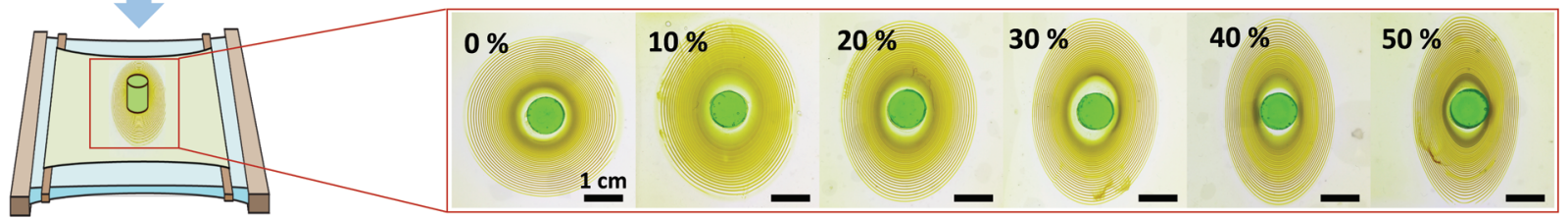

\section{B: Released (unloaded)}
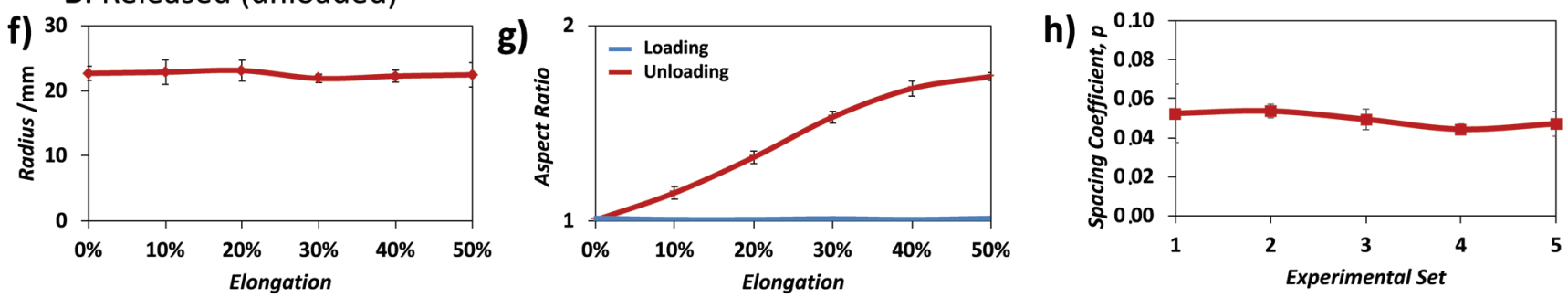

Figure 1. The effect of elastic deformation of the hydrogel on the simultaneous development of Liesegang patterns (LPs) in the gel. a) Growth patterns in

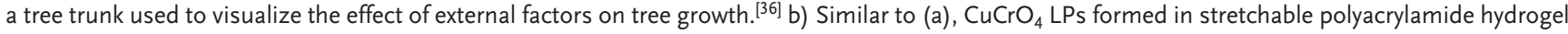
medium can be used to "record and read" the mechanical deformation in the medium. c) Schematic representation of the development of LPs in uniaxially loaded gels; after $24 \mathrm{~h}$ stretching "A" patterns are formed, which become "B" patterns when the gels are unloaded. d) Development of identical "A" patterns at different strains (0-50\%). The loading time is $24 \mathrm{~h}$ for all samples. e) Corresponding "B" patterns obtained upon unloading of gels with " $\mathrm{A}$ " patterns in (d). f) The outer radii of "A" patterns in (d), showing that the patterns developing under different \% strains are the same. g) Aspect ratio deviates from one upon unloading to obtain "B" patterns; the deviation from unity is more pronounced as the \% strain in the sample increases. Error bars in $(\mathrm{f})$ and $(\mathrm{g})$ correspond to standard deviations from six independent experiments. $h$ ) The "A" patterns are "Liesegang type" as verified by their constant spacing coefficient $(p)$ that is reproduced at each experimental set involving all elongation values (10-50\%). The outer electrolyte is $\mathrm{CuCl}_{2}$ (aq), $1.0 \mathrm{M}$ in an agarose stamp, and the inner electrolyte is $\mathrm{K}_{2} \mathrm{CrO}_{4}(\mathrm{aq}), 0.01 \mathrm{~m}$ in a polyacrylamide gel. For experimental details, see Supporting Information.

inputs, such as prestraining ${ }^{33]}$ (printing on a stretched substrate), that have been used for transformation of 2D structures into 3D objects. In this study, we show the formation of LPs in a stretchable polyacrylamide gel medium that retrieves information about the elastic deformation in the medium. Following the concurrent LP formation, information on the extent, duration, and time of elastic deformation can be obtained by "reading" the formed patterns, similar to the utilization of "growth rings" to estimate the weather and environmental conditions that affect tree growth (Figure 1a,b). The LP-gel system can function in 1D, $2 \mathrm{D}$, and 3D diffusion geometries; however, 3D diffusion geometries are rarely studied for artificial patterns due to the limitations in preparation of the gels in these geometries, ${ }^{[34,35]}$ and visualize mechanical deformation in these cases. The patterns can also be chemically erased to "re-record" further cycles of deformations. Finally, the relation between the mechanical deformation of the gel and the obtained pattern is quite reliable, such that the deformation can also be used to control the pattern formation, which can further be used to template lines of polypyrrole on the surfaces with different line spacing and widths.

In a typical formation of a LP in gel medium, a gel is doped with the outer electrolyte (a solution of one of the reactant salts of the precipitation reaction), and this gel is put into contact with another (acceptor) gel containing the inner electrolyte (a solution of the second reactant of the reaction). Usually, the acceptor gel is monitored for the formation of LPs as the outer electrolyte is diffusing into the acceptor gel since the outer electrolyte concentration is one or two orders of magnitudes higher 
than that of the inner electrolyte. Conventional gel materials used for developing LPs are agarose, gelatin, and silica gel, none of which can endure high compression or strains. To test our hypothesis that a mechanical input may affect LP formation in a gel, a mechanically robust and highly stretchable gel medium is needed, for which the mechanical input can be reversibly (elastically) applied. To accommodate the chemical reactiondiffusion system, the gel must be a hydrogel since water is the most common medium used for LP formation reactions. Recent reports on the chemical stability and mechanical behavior of stretchable polyacrylamide hydrogel materials ${ }^{[37,38]}$ guided us to choose this material in our experiments. In a typical experiment, we prepared the hydrogel solution with the addition of $0.01 \mathrm{M}$ $\mathrm{K}_{2} \mathrm{CrO}_{4}$ (inner electrolyte). Then, the solution was poured in a mold made of plexiglass sheets sized $7 \mathrm{~cm} \times 7 \mathrm{~cm} \times 2 \mathrm{~mm}$ and was allowed to gel for $24 \mathrm{~h}$ (see Figures S1-S3, Supporting Information, for details on the preparation of the gels with electrolytes). For the initial display of how LP formation can be affected by mechanical deformation; the prepared gels were transferred onto an Ecoflex substrate, which served to provide homogeneous elastic deformation. By applying uniaxial tensile stress ("loading"), we stretched the gels to $10-50 \%$, at these strains (up to $60 \%$ ) the gels are deformed elastically as reported in the literature ${ }^{[37]}$ and verified in our experiments. As shown in Figure 1c and Figure S4, Supporting Information, cylindrical agarose stamps $(1.0 \mathrm{~cm}$ diameter, $0.8 \mathrm{~cm}$ height) gels filled with $1.0 \mathrm{M} \mathrm{CuCl}_{2}$ (outer electrolyte) were applied at the centers of the stretched gels. Placing the stamp followed by careful stretching gives the same result. This wet stamping ${ }^{[39]}$ was allowed to set for $24 \mathrm{~h}$ to allow the development of patterns, which appeared as concentric rings, as expected. The stress was then released, and the gels were allowed to return to their original, unstretched shape ("unloading”). Upon mechanical loading for $24 \mathrm{~h}$ with a constant strain, the formation of LPs on the stretched gels were unaffected by the percent strain (10-50\%) (Figure 1d) and the positions of the Liesegang bands in the diffusion front were found to be identical in all samples (Figure 1f). The aspect ratio of the final rings remained equal to unity (Figure $1 \mathrm{~h}$ ). Since the hydrogels are $\approx 90 \%$ of water, the stretching of the medium only changes the gel geometry, concentration gradients, and the diffusion rates of the reactants, and hence the LP positions, remain unaffected. The effect of the percent strain on the formation of the LPs became visible only upon unloading (Figure 1e); after the stress was removed, and the gels returned to their original shapes, the aspect ratio of the final rings increased with the increasing percent strain (at the previous loading) of the gel and reached 1.7 for $50 \%$ strain (Figure 1g). A similar effect of uniaxial tension can also be seen in the "1D setup," where the LP bands forming under 10-50\% strain appear closer to the stamp when the samples are unloaded, as shown in Figure S5, Supporting Information. We should also note that the patterns formed during loading ("A" patterns) kept the Liesegang characteristics, meaning they have a constant spacing coefficient $(p)$ that is independent of the ring number (Figure 1h). The spacing coefficient was calculated as $X_{n+1} / X_{n}=1+p$, where $X_{n+1}$ and $X_{n}$ are the distance of the two consecutive bands measured from the junction point of the inner and outer electrolytes, respectively. This result showed that the applied mechanical field was quite homogeneous in the gel medium.
As shown in Figure 1, the effect of the applied mechanical field can be seen in the planar (2D) LP as concentric rings. Upon similar mechanical input, loading upon formation of LPs and then releasing (Figure 2a), in 1D gels Liesegang "bands" form (Figure 2b), and in 3D gels Liesegang "shells"[40] (Figure 2d) can be obtained for similar "A" patterns and "B" patterns upon loading and unloading, respectively. The 2D (Liesegang rings) and 3D (Liesegang shells) LP formations allow an understanding of the deformation process, either tension or compression, in different directions or on different planes located in various geometries with respect to the direction of the applied uniaxial stress. The effect of the mechanical deformation of the media on the LP is perhaps most vividly seen in the Liesegang shells, where anisotropy of pattern formation in the different planes, $X Z, Y Z$, and $X Y$, can be readily achieved by uniaxial compression (Figure $2 \mathrm{~d}$ ).

As shown above, the constant supply of mechanical energy (during mechanical loading) provides a change that affects the diffusion depth of the reactants in comparison to the diffusion in a nonstretched medium. In our experiments, this change became visual after unloading ("B" patterns), which provided the first noticeable difference in the overall LP formed in uniaxially elastically deformed gels, in comparison to the undeformed gels. A closer look at the patterns revealed a more interesting and emergent phenomenon that takes place upon the continuation of the reaction-diffusion after unloading the loaded samples. A new set of patterns is formed after a defined waiting period in the unloaded state (denoted as " $\mathrm{C}$ patterns" in Figure 2, and Movies S1 and S2, Supporting Information). We refer to this new set of patterns as post-deformation patterns or "post-patterns (PPs)" (Figure 2). PPs can be divided into two distinct parts, equally spaced patterns (EPs) and regular Liesegang patterns (LP2s). EPs form first after unloading the gel. Interestingly, through image analysis of the EPs (Figure 2e; for details on image analysis, see Figures S5-S7, Supporting Information), it was shown that these EPs do not have "Liesegang characteristics," they instead have constant spacing of consecutive bands (the spacing coefficient $(p+1)$ is close to unity) and constant band widths (Figure 2e). These characteristics differ from the well-known regularities of classical Liesegang patterns (spacing and width laws) introduced by Jablczynski and Droz. ${ }^{[41,42]}$ The transition from the LP1s to the EPs is quite abrupt compared to the timescale of diffusion, so the start of EP formation can be easily distinguished in the final overall pattern. As shown in Figure 2e, and Movie S1, Supporting Information, there is also a gradual subsequent reversion from the EPs to the LP2s in the later times of the poststress waiting period. The deformation of 2D and 3D samples gives a similar but visually more complicated output (Figure 2f). In 2D and 3D, there is also an anisotropy that arises from the differences in the response of the material for the load, in the direction of applied stress and other directions (Figure S7, Supporting Information). The formation of EPs can be explained by the instantaneous change in diffusion flux of the outer (invading) electrolyte due to the unloading of the gel, which governs the pattern formation behind the chemical front. Due to the diffusive nature of the front propagation in LPs, the spacing between the consecutive precipitation bands increases with increases in the widths of the bands. Immediately after unloading the gel, a steeper 


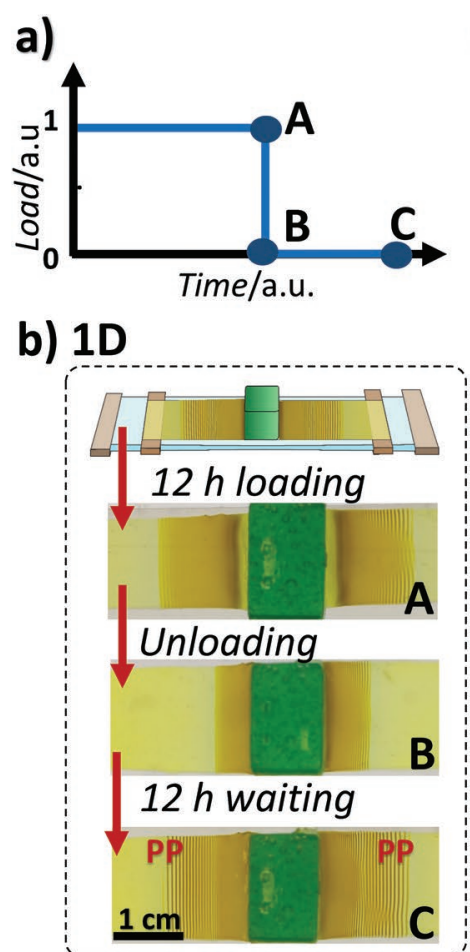

c) $2 \mathrm{D}$

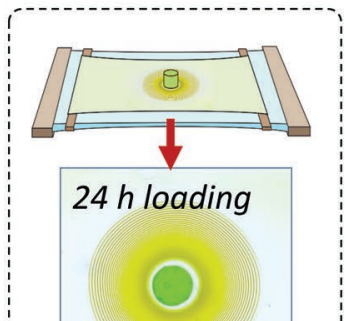

A

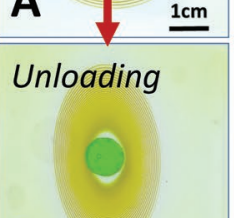

B

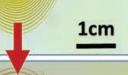

$18 \mathrm{~h}$ waiting

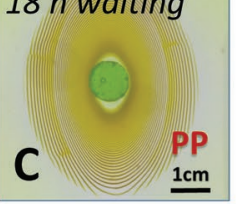

d) 3D

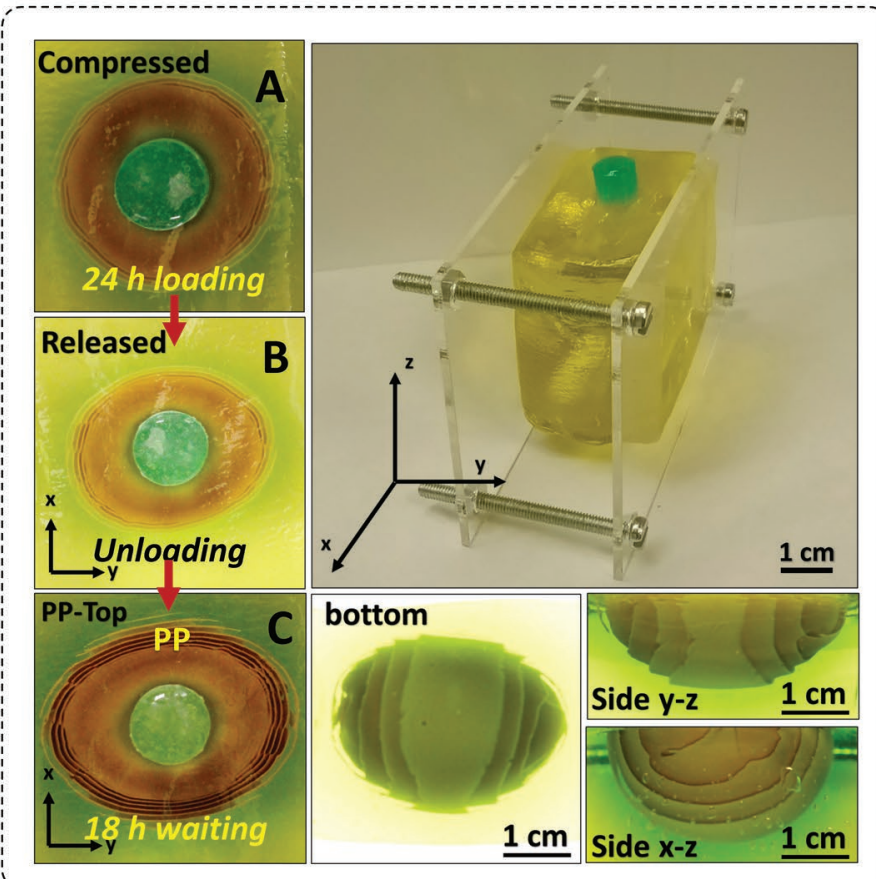

e)

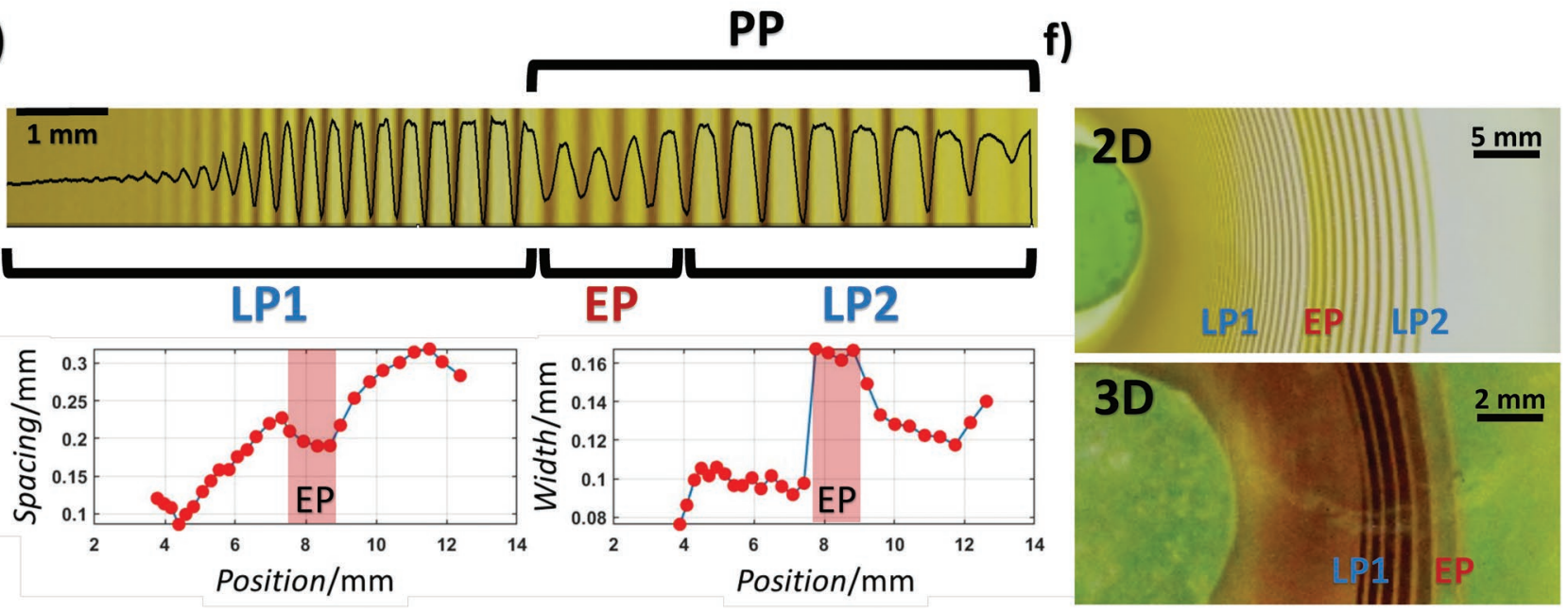

Figure 2. Formation of Liesegang patterns (LP1s) and post-patterns (PPs) in 1D, 2D, and 3D diffusion geometries in elastically deformed polyacrylamide hydrogels. PPs consist of two consecutive parts, equally spaced patterns (EPs) followed by regular Liesegang patterns (LP2s). a) The schematic representation of the mechanical deformation. b,c) "A" (loaded), "B" (unloaded), and "C" (unloaded, waiting) patterns in 1D, 2D, and 3D. In (b) and (c), uniaxial tension is applied to obtain $40 \%$ strain, and in d), $30 \%$ uniaxial compression in the $\gamma$-direction is applied on the gel cube as shown in (d). e) The gray value plot of the " $C$ " patterns in (b), and their spacing and width analyses. f) Close-up of " $C$ " patterns in (c) and (d). See also Movies S1 and S2, Supporting Information, for "live images" of the pattern development. The outer electrolyte is $\mathrm{CuCl}_{2}$ (aq), $1.0 \mathrm{M}$ in an agarose stamp and the inner electrolyte is $\mathrm{K}_{2} \mathrm{CrO}_{4}(\mathrm{aq}) 0.01 \mathrm{~m}$ in a polyacrylamide gel; for $3 \mathrm{D}$, polyacrylamide-sodium alginate is the gel. For experimental details, see Supporting Information.

diffusion gradient of the outer electrolyte forms, causes a transient increase in the speed of the front with a constant velocity, which produces the EPs. It has been shown that precipitation coupled to a chemical front moving with a constant velocity produces equidistant bands with a constant width, as seen in Figure 2e. ${ }^{[43]}$ Once the chemical front slows down due to diffusion, the LP2s start to appear with Liesegang characteristics (increasing spacing and widths of the bands).
As mentioned in the introduction, the LPs can be affected and controlled by many internal (e.g., concentrations of the reactants) or external (e.g., an electrical field) changes. However, in all cases, the formed patterns were all of the Liesegang type, although some secondary patterns may also be observed in the media. ${ }^{[4]}$ Therefore, this is the first report of divergence from Liesegang character upon application of an external (mechanical) input. The reason for this difference from previous reports 

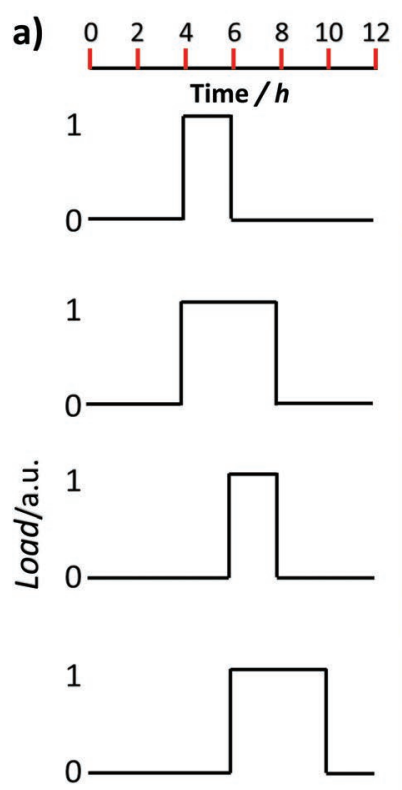

1

0 b)

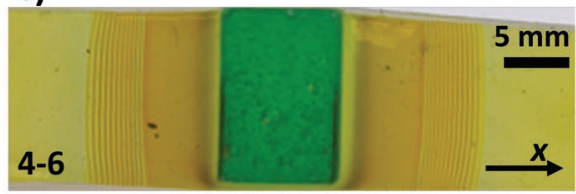

4-8
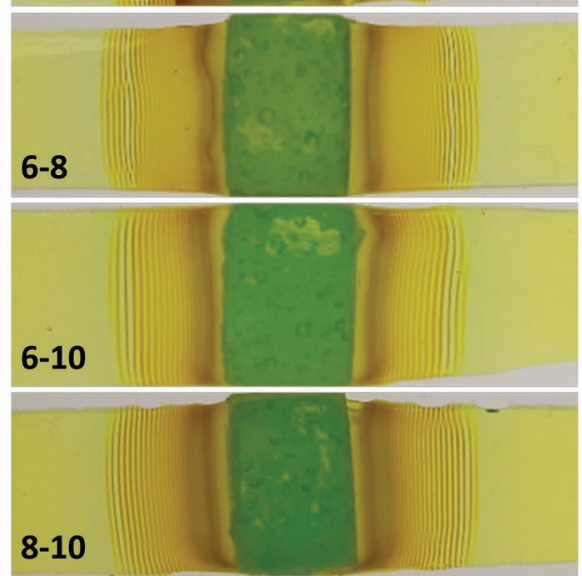

c)
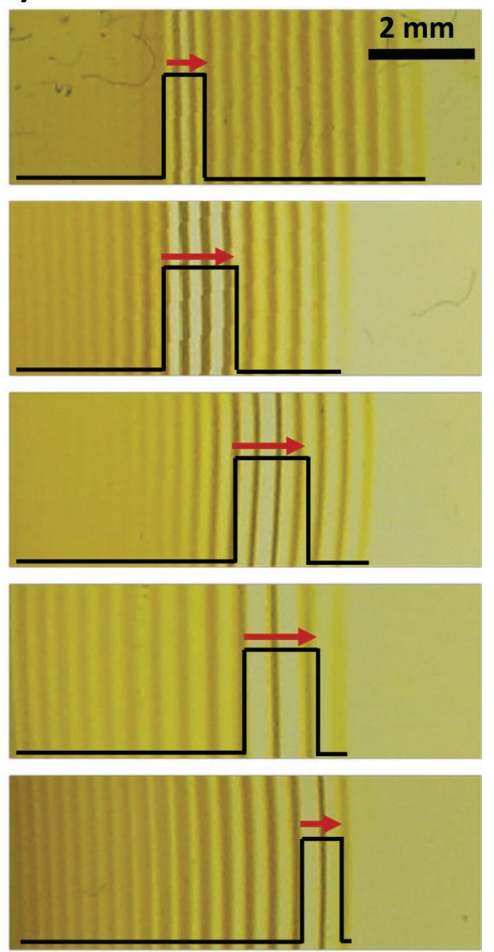

Figure 3. Tracking the time and duration of mechanical deformation pulses with LPs. a) The pulse applied on the gels in the 1D setup upon LP formation under identical chemical conditions (the total time of the experiment is $12 \mathrm{~h}$ ). 0 and 1 correspond to unstretched and stretched states, respectively. b) Views of the LP patterns for each case after a total of $12 \mathrm{~h}$. c) LPs shown in (b) reflect on the different start times of the pulse as the beginning of a lighter colored region in the LPs, different durations of the pulse with the different numbers of PPs formed. The outer electrolyte is $\mathrm{CuCl}_{2}(\mathrm{aq}), 1.0 \mathrm{M}$ in an agarose stamp and the inner electrolyte is $\mathrm{K}_{2} \mathrm{CrO}_{4}$ (aq) $0.01 \mathrm{M}$ in a polyacrylamide gel. For experimental details, see Supporting Information.

and the emergent complexity is that in this study diffusion (a nonequilibrium phenomenon) is perturbed by a "pulsated" input, which is achieved by the unloading process.

Since the LP to PP transition upon unloading was visually clear in our experiments, we probed its usability for tracking the time of the mechanical deformation. For this purpose, we started with a simple geometry, namely, with 1D samples prepared in identical geometries and the same set of electrolytes. We applied a cyclic load to these samples, where full "pulses" of mechanical deformation of 2 and $4 \mathrm{~h}$ (to achieve $40 \%$ strain for all samples) were started at different points in time $(2,4,6$, and $8 \mathrm{~h}$ after diffusion starts) (Figure 3a). A step-by-step scheme of this pulse can also be seen in Figure S8, Supporting Information. Experimental photos of the final patterns obtained after a constant total time of $12 \mathrm{~h}$ for each run (Figure 3b,c) show that: 1) as the start time of the given pulse increases, the PPs start at a later order of bands, and 2) as the duration of the load pulse increases for the same start time, the number of observed PPs increase. The visual information written in the post-patterns can also be detailed, modeled, and augmented by calculations (Figure S9, Supporting Information), which provide a precise quantitative explanation of the systems. The "tracking" of the PPs to retrieve information about the mechanical deformation can be performed for the 2D (Figure S10, Supporting Information) and $3 \mathrm{D}$ diffusion geometries, with an additional set of information for the direction of the uniaxial tension and compression, respectively.
The patterns formed in hydrogels are completely chemically erasable and reversible (note the elastic deformation itself is "mechanically reversible," too), which allows the gels to be used in consecutive cycles of patterning and deformation. In our experiments, the clarity and the mechanical properties of the gels were retained completely even after tens of cycles (Figure 4a). The well-defined differences in pattern formation under various load pulses display an unprecedented control over the final architectures for tracking the time, the duration, and in the case of the $2 \mathrm{D}$ and $3 \mathrm{D}$ geometries, the direction of the environmental changes in the reaction-diffusion media. It is apparent that by planning the mechanical pulse input, numerous possibilities of precipitate patterns with high complexity can be achieved in the gels. It is also possible to pattern other materials using previously obtained periodic patterns. To display this, we polymerized pyrrole and deposited the resulting polypyrrole on the patterns by using the $\mathrm{CuCrO}_{4}$ bands previously formed in a 1D setup. Polypyrrole is the most common electrically conductive polymer that has a wide range of applications in responsive materials, electronics, and chemical sensors which has recently also expanded into hydrogels. ${ }^{[45]}$ Recently, polypyrrole has been used in hydrogels for various functions, for example, to make multiresponsive materials. ${ }^{[46]}$ Soft actuators for soft robots can also be made using polypyrrole. ${ }^{[47]}$ In all these applications, complex patterning of polypyrrole might be desirable for achieving new levels of complexity. Among many possibilities, we chose polypyrrole as an 


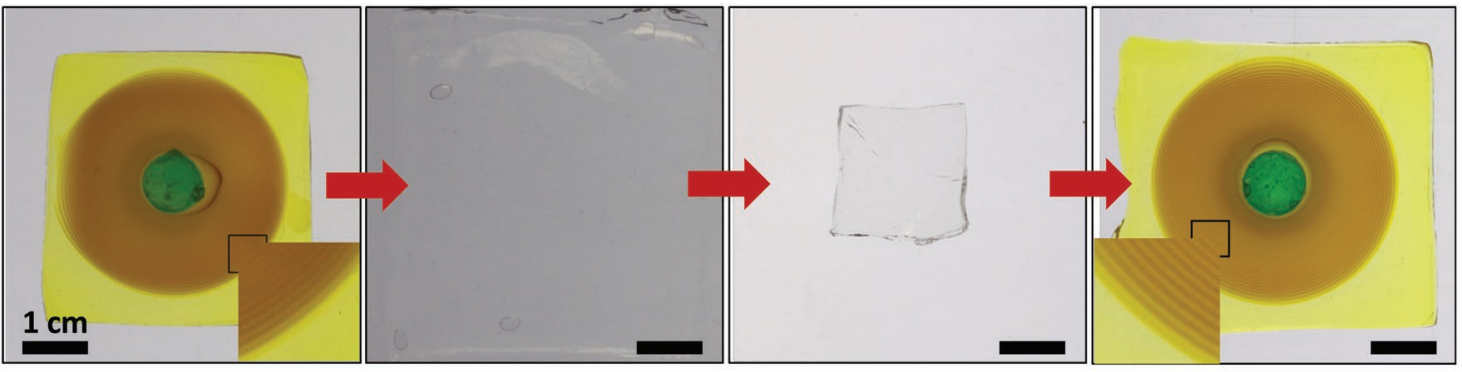
First
Pattern
patterning
removal
Drying
Second
patterning
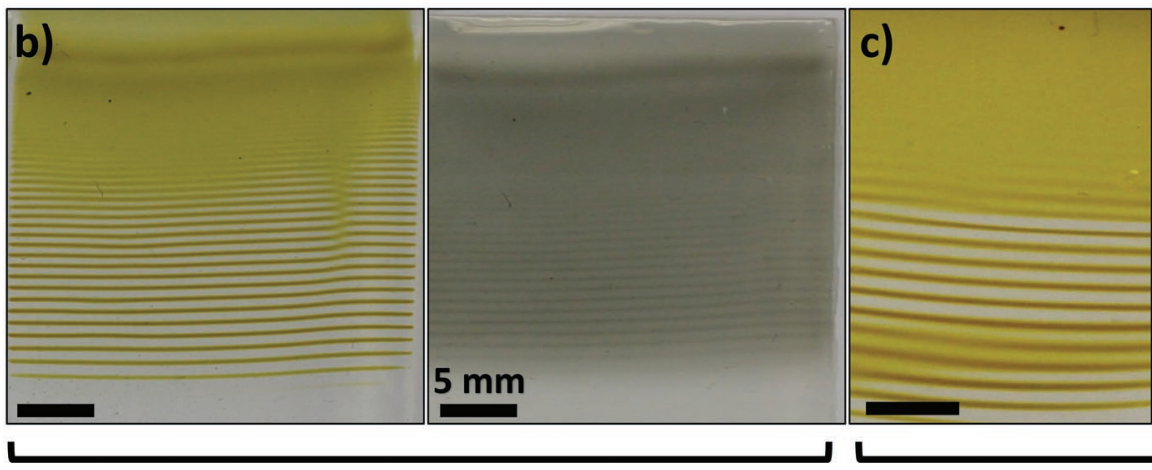

No mechanical deformation
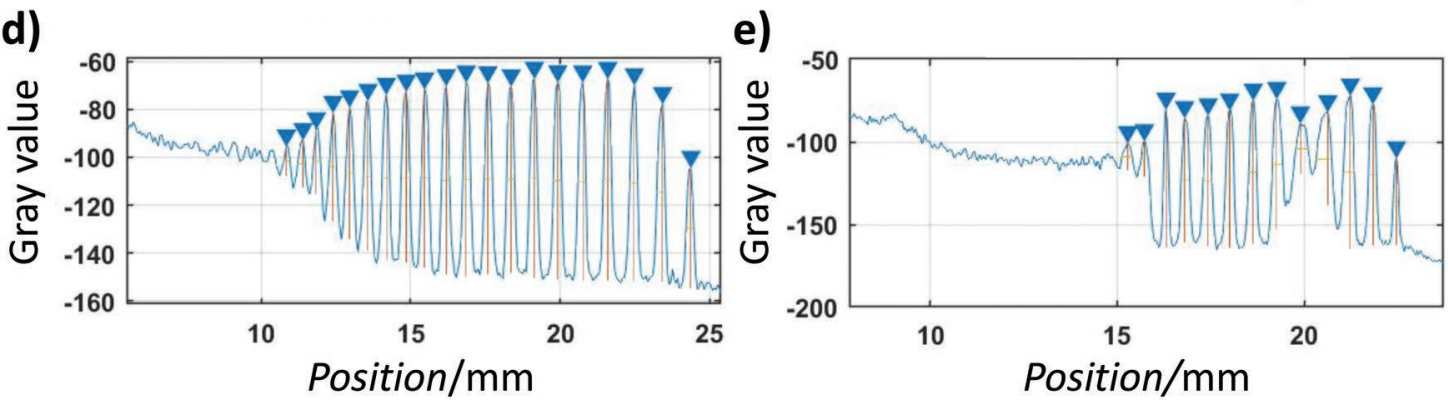

Figure 4. Rewriting of LPs in PAA hydrogels and the complex patterning of polypyrrole on the precipitation bands. a) Pattern erasing by dipping the gel in $0.1 \mathrm{M} \mathrm{HCl}$ solution, and second patterning after washing and drying of the first. b) Polymerization of pyrrole on $\mathrm{CuCrO}_{4}$ patterns by dipping the samples in $30 \mathrm{~mL}$ pyrrole solution $(0.1 \mathrm{M})$ and $\mathrm{HCl}(1 \mathrm{~mm})$ for $4 \mathrm{~h}$. c) Complex patterning of polypyrrole by elastic deformation with a start time of $6 \mathrm{~h}$ the duration of $2 \mathrm{~h}$, and with $40 \%$ elongation of the gel. The total time for the pattern development is $12 \mathrm{~h}$. d,e) Gray value analysis of the patterns in (b) and (c) showing differences in the lines spacings. For initial $\mathrm{CuCrO}_{4}$ patterns the outer electrolyte is $\mathrm{CuCl}_{2}(\mathrm{aq}), 1.0 \mathrm{M}$ in an agarose stamp and the inner electrolyte is $\mathrm{K}_{2} \mathrm{CrO}_{4}(\mathrm{aq}), 0.01 \mathrm{M}$ in a polyacrylamide gel. For experimental details, see Supporting Information.

example of a material that can be patterned since the polymerization of pyrrole has matching chemistry with the patterns in this study. $\mathrm{Cu}^{2+}$ obtained from the patterned precipitate can be used as an initiator in this reaction. In the experiment, $4 \mathrm{~h}$ after the introduction of the monomer $(3.0 \mathrm{~mm}, 0.2 \mathrm{~g})$ onto the surface of the gel, polypyrrole only formed on top of the bands where the $\mathrm{Cu}^{2+}$ concentration is the highest (after washing the gel with water). $\mathrm{Cu}^{2+}$, which was used as the polymerization initiator, was obtained by applying a minute amount of $\mathrm{HCl}$ onto the previously water-rinsed gel with $\mathrm{CuCrO}_{4}$ precipitation bands. For details of the polymerization procedure of pyrrole on the patterns, see Supporting Information. Of the two prepared samples, the sample formed using the bands formed upon mechanical input (40\% strain, $24 \mathrm{~h}$ loading, Figure 4c) is distinctly different from the sample without any mechanical deformation (Figure $4 \mathrm{~b}$ ), with respect to the aspect ratio of the overall pattern and the ring distances (Figure 4d,e; Figure S11, Supporting Information), as expected.

The formation and appearance of periodic precipitation patterns can be affected and manipulated by the mechanical input exerted on the gel media in which the patterns are developing. We have shown that it is possible to obtain information on the start time, the duration, and the direction of mechanical input in samples with 1D, 2D, and 3D diffusion geometries. We believe that the concepts presented here can be used in various platforms, for example, to monitor diffusion and predict deformation under pressure in the precipitation patterns in sedimentary rocks. With some further development, the idea can be extended to retrieve information about long-duration plastic deformation, for example, in buildings. This concept 
can also be used in probing fatigue in soft materials, especially materials that are subjected to cycles of elastic deformation, such as in soft robots. We also showed that the mechanically controlled formation of the geometries of the $\mathrm{CuCrO}_{4}$ precipitate bands can be used to deposit polypyrrole in complex patterns, on differently spaced bands. We believe these initial experiments can be elaborated with some planning and further optimization, and the precise interplay between the mechanical inputs can be used to design and pattern other synthetically valuable products, opening new possibilities for the complex patterning of other materials with different chemistries through nonequilibrium systems.

\section{Supporting Information}

Supporting Information is available from the Wiley Online Library or from the author.

\section{Acknowledgements}

This work was supported by TÜBITAK under award number $116 Z 116$ and by the National Research, Development and Innovation Office of Hungary (NN125752 and TÉT_15-1-2016-0079) and the BME-Nanotechnology FIKP grant of EMMI (BME FIKP-NAT). B.B. gratefully acknowledges the support from BAGEP 2016 (Science Academy Young Scientists Program) award.

\section{Conflict of Interest}

The authors declare no conflict of interest.

\section{Keywords}

Liesegang phenomena, nonequilibrium systems, periodic patterns, polyacrylamide gel, reaction-diffusion, stretchable gels

Received: September 4, 2019

Revised: November 19, 2019

Published online: January 3, 2020

[1] Z. Tan, S. Chen, X. Peng, L. Zhang, C. Gao, Science 2018, 360, 518.

[2] A. F. Taylor, M. R. Tinsley, Nat. Chem. 2009, 1, 340.

[3] L. Cera, C. A. Schalley, Adv. Mater. 2018, 30, 1707029.

[4] T. Shinbrot, F. J. Muzzio, Nature 2001, 410, 251.

[5] R. M. Walliser, F. Boudoire, E. Orosz, R. Tóth, A. Braun, E. C. Constable, Z. Rácz, I. Lagzi, Langmuir 2015, 31, 1828.

[6] S. Sadek, R. Sultan, Precip. Patterns React.-Diffus. Syst. 2010, 661, 1.

[7] R. E. Liesegang, Naturwiss. Wochenschr. 1896, 11, 353.

[8] E. Ramírez-Álvarez, F. Montoya, T. Buhse, W. Rios-Herrera, J. Torres-Guzmán, M. Rivera, G. Martínez-Mekler, M. F. Müller, Sci. Rep. 2016, 6, 23402.

[9] B. A. Grzybowski, Chemistry in Motion, Wiley, New York 2016.
[10] R. Matalon, A. Packter, J. Colloid Sci. 1955, 10, 46.

[11] Z. Rácz, Phys. A 1999, 274, 50.

[12] L. Badr, R. Sultan, J. Phys. Chem. A 2009, 113, 6581.

[13] I. Lagzi, Langmuir 2012, 28, 3350.

[14] Z. Shreif, L. Mandalian, A. Abi-Haydar, R. Sultan, Phys. Chem. Chem. Phys. 2004, 6, 3461.

[15] A. Toramaru, T. Harada, T. Okamura, Phys. D 2003, 183, 133.

[16] E. Kárpáti-Smidróczki, A. Büki, M. Zrínyi, Colloid Polym. Sci. 1995, $273,857$.

[17] S. K. Smoukov, A. Bitner, C. J. Campbell, K. Kandere-Grzybowska, B. A. Grzybowski, J. Am. Chem. Soc. 2005, 127, 17803.

[18] R. Makki, M. Al-Ghoul, R. Sultan, J. Phys. Chem. A 2009, 113, 6049.

[19] I. Lagzi, Phys. Chem. Chem. Phys. 2002, 4, 1268.

[20] M. Al-Ghoul, R. Sultan, J. Phys. Chem. A 2003, 107, 1095.

[21] I. Bena, M. Droz, Z. Rácz, J. Chem. Phys. 2005, 122, 204502.

[22] Y. Kanazawa, Y. Asakuma, J. Cryst. Process Technol. 2014, 4, 65.

[23] D. A. Smith, J. Chem. Phys. 1984, 81, 3102.

[24] G. T. Dee, Phys. Rev. Lett. 1986, 57, 275.

[25] H. J. Krug, H. Brandtstädter, J. Phys. Chem. A 1999, 103, 7811.

[26] T. Antal, M. Droz, J. Magnin, Z. Rácz, Phys. Rev. Lett. 1999, 83, 2880.

[27] H. Yan, Y. Zhao, C. Qiu, H. Wu, Sens. Actuators, B 2008, 132, 20.

[28] M. K. Beyer, H. Clausen-Schaumann, Chem. Rev. 2005, 105, 2921.

[29] H. T. Baytekin, B. Baytekin, B. A. Grzybowski, Angew. Chem., Int. Ed. 2012, 51, 3596.

[30] B. Baytekin, H. T. Baytekin, B. A. Grzybowski, Angew. Chem., Int. Ed. 2014, 53, 6946.

[31] H. T. Baytekin, B. Baytekin, S. Huda, Z. Yavuz, B. A. Grzybowski, J. Am. Chem. Soc. 2015, 137, 1726.

[32] C. R. Hickenboth, J. S. Moore, S. R. White, N. R. Sottos, J. Baudry, S. R. Wilson, Nature 2007, 446, 423.

[33] W. Bai, Z. Wang, H. Jang, Y. Xu, H. Luan, Y. Ma, X. Wang, F. Zhang, X. Shan, X. Guo, Y. Zhang, D. Choi, T. Xie, K. Nan, N. Zheng, Y. Huang, B. H. Kim, Y. Zhang, J. A. Rogers, R. Sun, P. Kohli, Z. Xu, J. Ye, Q. Zhang, Z. Xie, X. Feng, Adv. Mater. 2018, 31, 1805615.

[34] H. Zhang, K. Zhan, Y. Chen, G. Chen, X. Zhou, J. Liu, M. Wu, H. Ni, J. Sol-Gel Sci. Technol. 2014, 71, 597.

[35] M. Ripszám, Á. Nagy, A. Volford, F. Izsák, I. Lagzi, Chem. Phys. Lett. 2005, 414, 384.

[36] R. B. Hoadley, Understanding Wood: A Craftsman's Guide to Wood Technology, Taunton Press, Newtown, CT, USA 1980.

[37] J. Y. Sun, X. Zhao, W. R. K. Illeperuma, O. Chaudhuri, K. H. Oh, D. J. Mooney, J. J. Vlassak, Z. Suo, Nature 2012, 489, 133.

[38] P. Calvert, Adv. Mater. 2009, 21, 743.

[39] I. T. Bensemann, M. Fialkowski, B. A. Grzybowski, J. Phys. Chem. B 2005, 109, 2774.

[40] L. Badr, Z. Moussa, A. Hariri, R. Sultan, Phys. Rev. E 2011, 83, 016109.

[41] C. K. Jablczynski, Bull. Soc. Chim. Fr. 1923, 33, 1592.

[42] M. Droz, J. Magnin, M. Zrinyi, J. Chem. Phys. 1999, 110, 9618.

[43] I. Lagzi, D. Kármán, Chem. Phys. Lett. 2003, 372, 831.

[44] S. K. Smoukov, I. Lagzi, B. A. Grzybowski, J. Phys. Chem. Lett. 2011, 2, 345 .

[45] D. Mawad, A. Lauto, G. G. Wallace, in Polymeric Hydrogels as Smart Biomaterials, Springer Series on Polymer and Composite Materials (Ed: S. Kalia), Springer, New York 2016.

[46] H. Kim, K. Kim, S. J. Lee, NPG Asia Mater. 2017, 9, e445.

[47] A. Benouhiba, K. Rabenorosoa, P. Rougeot, M. Ouisse, N. An-dreff, in 2018 IEEE/RSJ Int. Conf. on Intelligent Robots and Systems (IROS), IEEE, Piscataway, NJ, USA 2018, pp. 7500-7506. 